\title{
Algorithms for Sparse Gaussian Elimination With Partial Pivoting
}

\author{
ANDREW H. SHERMAN \\ The University of Texas at Austin
}

\begin{abstract}
We compare several algorthms for sparse Gaussian elimination with column interchanges. The algorithms are all denved from the same basic elimination scheme, and they differ mainly in implementation details. We examine their theoretical behavior and compare their performances on a number of test problems with that of a high quality complete threshold pivoting code. Our conclusion is that partial pivoting codes perform quite well and that they should be considered for sparse problems whenever pivoting for numencal stability is required.
\end{abstract}

Key Words and Phrases: sparse Gaussian elimination, sparse linear systems, linear equations, analysis of algorithms, pivoting algorithms

CR Categories: 4.6, 5.14, 5.25

The Algorithm NSPIV, A Fortran Subroutine for Sparse Gaussian Elımination With Partial Pivoting. ACM Trans. Math. Software 4, 4 (Dec 1978), 391-398.

\section{INTRODUCTION}

Let $A$ be an $N \times N$ nonsingular matrix, and consider the system of linear equations

$$
A x=b,
$$

where $x$ and $b$ are vectors of length $N$. If $N$ is small or $A$ is dense (i.e. most entries $a_{\imath \jmath}$ are nonzero), then the standard algorithm for solving eq. (1.1) is Gaussian elimination with partial pivoting (cf. Forsythe and Moler [6]). This algorithm is quite well understood, from both the theoretical and practical points of view, and it produces an accurate solution $x$ (provided that $A$ is well conditioned and the equations are well scaled, both of which we shall assume throughout).

When $N$ is large and the matrix $A$ is sparse (i.e. most entries $a_{v}$ are zero), the situation is quite different. Now there are two problems to contend with: the accurate computation of $x$, and the exploitation of the large number of zeros in $A$

Permission to copy without fee all or part of this material is granted provided that the coples are not made or distributed for direct commercial advantage, the ACM copyright notice and the title of the publication and its date appear, and notice is given that copying is by permission of the Association for Computing Machinery. To copy otherwise, or to republish, requires a fee and/or specific permission.

This work was supported in part by the National Science Foundation under Grant NSF DCR-7307998 and in part by the Energy Research and Development Administration under Grant US ERDA E(11-1) 2383. An earlier version of this paper was presented at the 1976 SIAM National Meeting. This work was performed while the author was on the faculty of the Department of Computer Scrence, Unıversity of Illinois at Urbana-Champaign.

Author's address: Department of Computer Sciences, Painter 328, The University of Texas at Austin, Austin, TX 78712.

(C) 1978 ACM 0098-3500/78/1200-0330 $\$ 00.75$ 
to reduce storage and computing time. When $A$ is symmetric and positive definite, a number of methods may be effective, including variants of Gaussian elimination and iterative methods such as conjugate gradient. However, in most other cases, Gaussian elimination with pivoting seems to be the best method currently available.

In this paper we examine algorithms for sparse Gaussian elimination with partial pivoting. In Section 2 we introduce a basic high-level algorithm and point out several problems that arise in practice. In Section 3 we discuss several possible algorithm refinements which solve the practical problems, and we give a theoretical comparison of them based on their behavior in the best, average, and worst cases. Unfortunately, we do not believe that our theoretical analysis reflects what happens in practice, so in Section 4 we give the results of some numerical experiments with our algorithms and compare them with one of the better existing codes for Gaussian elimination with pivoting. Finally, we conclude in Section 5 by suggesting directions for future development based on the results in this paper.

\section{A BASIC ALGORITHM}

In this section we examine a basic algorithm for sparse Gaussian elimination with partial pivoting. More precisely, we consider an algorithm which performs Gaussian elimination with column interchanges on the matrix $\boldsymbol{A}$ to effectively obtain a factorization of the form

$$
A Q=L U
$$

where $L$ is lower triangular, $U$ is unit upper triangular, and $Q$ is a permutation matrix corresponding to the column interchanges. Once this factorization has been obtained, we can compute $x$ by solving the systems

$$
L y=b \text { and } U Q^{\mathrm{T}} x=y,
$$

although we will not discuss this forward and back solution process here. The algorithm we give is a modification of an algorithm without pivoting discussed in [5].

The basic algorithm, given in Figure 1, is a row-oriented version of Gaussian elimination with column interchanges. It consists of $N$ steps, during each of which one row of $A$ is processed. When processing the $k$ th row at the $k$ th step, $I_{k}$ is used to hold the indices of all columns containing nonzeros in the $k$ th row. Initially (line 2), these are just the nonzero columns of $A$ in the $k$ th row. Then, in increasing order, for each $m \in I_{k}, m<k$, a multiple of row $\tilde{m}$ of $U$ is subtracted from row $k$ to annihilate $a_{k m}$ (lines 3-8). This may cause fill-in, i.e. the introduction of new nonzero elements in the $k$ th row, so $I_{k}$ must be updated (line 5). Finally, when all $m<k$ have been processed, $I_{k}$ contains the indices of columns which contain nonzeros in the portion of the $k$ th row lying in the upper triangle of $U$. If $A$ is nonsingular, $I_{k}$ will not be empty, so that $m \leq N$ will hold in line 9 . In that case the algorithm selects the remaining nonzero element with maximum modulus and interchanges its column with the $k$ th column. If $A$ is singular, on the other hand, then either $m=N+1$ in line 9 , or $a_{k l}=0$ in line 11. In either case, the algorithm takes a failure exit. (However, this situation could be handled simply by storing a null $k$ th row, if one wished to deal with singular matrices.) 


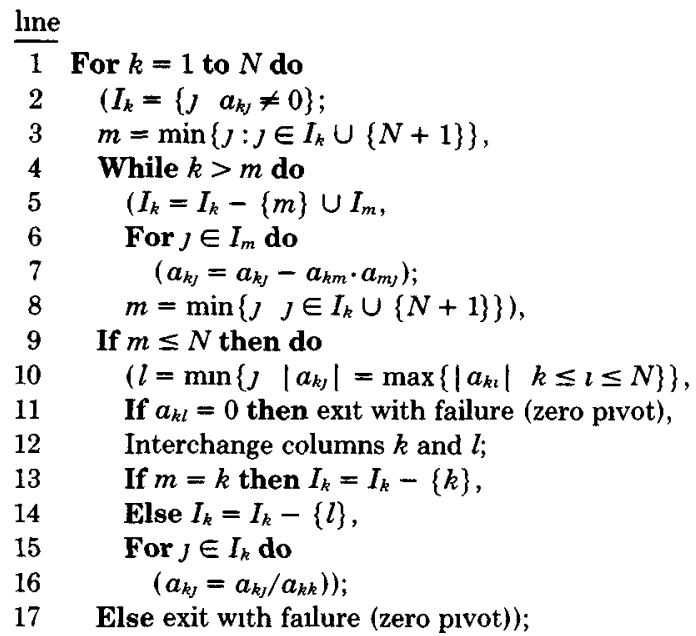

Fig. 1 Algorithm 1

That Algorithm 1 is numerically stable can be shown quite easily by relating it to the application of standard Gaussian elimination with row interchanges to $A^{\mathrm{T}}$. In fact, assume that such a procedure produces a factorization of $A^{\mathrm{T}}$ as

$$
P A^{\mathrm{T}}=\tilde{L} \tilde{U}
$$

where $\tilde{L}$ is unit lower triangular, $\tilde{U}$ is upper triangular, and $P$ is a permutation matrix corresponding to the row interchanges. Then we can show that Algorithm 1 produces the factorization (2.1) of $A$ with $L=\tilde{U}^{\mathrm{T}}, U=\tilde{L}^{\mathrm{T}}$, and $Q=P^{\mathrm{T}}$. Since the computation of eq. (2.2) is numerically stable (cf. [6]), that of eq. (2.1) by Algorithm 1 is also.

In Algorithm 1 there are only two operations whose costs can be greatly affected by the column interchanges due to pivoting: the selection of $m$ in lines 3 and 8 , and the updating of $I_{k}$ in line 5 . The costs of these operations may depend critically on the representation of the sets $I_{k}$ and $I_{m}$ (e.g ordered versus unordered, list versus array), while the costs of other operations in the algorithm in general do not.

As an illustration, consider two possible representations of $I_{k}$ : as a linked list in increasing order, and as a heap with smaller nodes nearer the root (cf. Knuth [7, p. 145]). In the first case, selecting $m$ is easy, since we want to choose the first entry of $I_{k}$, and the updating operation in line 5 can be performed as a linear merge of the two ordered lists in $O\left(\left|I_{m}\right|+\left|I_{k}\right|\right)$ time. (We use $\left|I_{m}\right|$ and $\left|I_{k}\right|$ to denote the number of entries in $I_{m}$ and $I_{k}$, respectively, when we start to merge $I_{m}$ into $I_{k}$ ) In the second case, selecting $m$ is still easy, since we want to choose the root of the heap, but now the updating operation may be more costly, since we must delete $m$ and separately insert each entry of $I_{m}$ into the heap for $I_{k}$. This may require a total of $O\left(\left|I_{m}\right| \log _{2}\left(\left|I_{m}\right|+\left|I_{k}\right|\right)\right)$ time.

From this example it might seem that there is no question as to the proper course: simply use an ordered linked list representation for $I_{k}$ (and an ordered array representation for previously computed sets $I_{m}$ ). In fact, if there were no pivoting, this conclusion would probably be correct. However, as soon as column 
interchanges can occur, the situation is not so clear cut. The problem is that while $I_{m}$ was in increasing order when it was computed at the $m$ th step, subsequent pivoting operations may cause it to be out of order at the $k$ th step. Thus the set union operation can no longer be performed as a simple ordered list merge. We might try to avoid this problem by keeping the sets $I_{k}$ in some canonical order (say the initial column order of $A$ ), but then the selection of $m$ would become difficult, since it would require a (necessarily linear) search for the minimum remaining column index in $I_{k}$ (in the current column order) at the $k$ th step.

In the remainder of this paper, we use Algorithm 1 as the basis for our discussion of various alternative means of implementing the selection and updating operations which seem so problematical when pivoting is permitted. The next section contains descriptions of several possible implementations and a limited theoretical discussion of them. In Section 4, we present more meaningful comparisons, based on numerical experiments.

\section{ALGORITHM IMPLEMENTATIONS}

In this section we present six different ways of implementing the selection and updating operations of Algorithm 1. For each we briefly describe the representations used for $I_{k}$ and $I_{m}$ (i.e. for $I_{k}$ during the $k$ th step and for the previously computed sets $I_{m}$ ). Then we outline the methods used to select $m$ and to form the union $I_{k} \cup I_{m}$, giving rough estimates of the costs of these operations in the best case (no pivoting), the average case, and the worst case. By average case here, we mean the average case with respect to the $N$ ! possible reorderings of the columns of $A$, not with respect to all possible matrices $A$. While the former may be less interesting, the latter seems all but impossible to study except through some (probably unrealistic) testing program using randomly generated matrices $A$.

\section{A. Run Insertion}

Each set $I_{k}$ is kept as a linked list in increasing order with respect to the current column order at the $k$ th step, and the sets $I_{m}$ are kept as arrays in increasing order with respect to the column order at the $m$ th step, where they were computed. The selection of $m$ requires constant time, since $m$ is always the first remaining entry of $I_{k}$. To form $I_{k} \cup I_{m}$, we effectively split $I_{m}$ into its component increasing runs (cf. Knuth [7, p. 34]) and separately merge each of the runs into $I_{k}$. In practice, this is done by merging the entries of $I_{m}$ into $I_{k}$ and backing up to the beginning of $I_{k}$ whenever an out-of-order entry is found in $I_{m}$. In the best case, the union operation requires $O\left(\left|I_{m}\right|+\left|I_{k}\right|\right)$ time, and in both the average and worst cases it requires $O\left(\left|I_{m}\right| \cdot\left|I_{k}\right|\right)$ time (cf. Knuth [7, pp. 34-45], for a discussion of the average number of runs in a sequence).

\section{B. List Merge with Bubble Sort}

The sets $I_{k}$ and $I_{m}$ are kept as in implementation A, except that $I_{m}$ is reordered whenever it is used. As before, choosing $m$ requires constant time. To form $I_{k} \cup I_{m}$, we first sort $I_{m}$ with a bubble sort (cf. Knuth [7, pp. 106-110]) and then perform an ordered list merge. In the best case, this will require $O\left(\left|I_{m}\right|+\left|I_{k}\right|\right)$ time, while in the average and worst cases it will require $O\left(\left|I_{m}\right|^{2}+\left|I_{k}\right|\right)$ time. 


\section{List Merge with Insertion Sort}

This is identical with implementation B, except that $I_{m}$ is sorted with a straight insertion sort (cf. Knuth [7, pp. 80-82]) when it is used. As above, $m$ can be chosen in constant time, and the set union operation requires $\left.O\left|I_{m}\right|+\left|I_{k}\right|\right)$ time in the best case and $O\left(\left|I_{m}\right|^{2}+\left|I_{k}\right|\right)$ time in the average and worst cases.

\section{List Merge with Shell Sort}

This is also identical with implementation B, except that $I_{m}$ is sorted with a Shell sort (cf. Knuth [7, pp. 84-95]) using increments as suggested in Knuth [7, p. 95]. Again $m$ can be chosen in constant time, and the set union operation requires $O\left(\left|I_{m}\right|^{2}+\left|I_{k}\right|\right)$ time in the worst case. In the average case, however, only $O\left(\left|I_{m}\right|^{3 / 2}+\left|I_{k}\right|\right)$ time is required for $\left|I_{m}\right| \leq 121$.

\section{E. Heap Insertion}

The set $I_{k}$ is split into two parts: $I 1_{k}=\left\{m \in I_{k}: m \leq k\right\}$ and $I 2_{k}=\left\{m \in I_{k}\right.$ : $m>k\} . I 1_{k}$ is kept as a heap with smaller nodes nearer the root (cf. Knuth [7, p. $145]$ ), and $I 2_{k}$ is kept as an unordered array. In addition, the characteristic vector of $I_{k}$ (cf. Aho et al. [1, p. 49]) is kept to avoid storing duplicate entries. At the end of the $k$ th step, $I 1_{k}$ is empty, and $I_{k}=I 2_{k}$. Hence we assume that the sets $I_{m}$ are stored as unordered arrays. (In fact, there is no loss in not having the sets $I_{m}$ ordered.) Choosing $m$ in this case still requires constant time, since we want to choose the root of the heap for $I 1_{k}$. To form $I_{k} \cup I_{m}$, we separately add each entry of $I_{m}$ either to the heap for $I 1_{k}$ or to the end of the unordered array for $I 2_{k}$. To insert an entry into the heap requires $O\left(\log _{2}\left|I 1_{k}\right|\right)$ time, and to add an entry to the unordered array requires constant time. Thus we expect that the best case time for the set union operation will be $O\left(\left|I_{m}\right|\right)$ time (when all the entries of $I_{m}$ are larger than $k)$, and that the average and worst case times will be $O\left(\left|I_{m}\right| \log _{2}\right.$ $\left.\left(\left|I_{m}\right|+\left|I_{k}\right|\right)\right)$. Note that deleting $m$ from $I_{k}$ requires $O\left(\log _{2}\left|I_{k}\right|\right)$ time, a usually insignificant cost.

\section{F. Pivot Search (cf. Curtis and Reıd [3])}

The set $I_{k}$ is kept as a linked list in increasing order with respect to a canonical ordering (e.g. the initial column order of $A$ ), and the sets $I_{m}$ are kept as ordered arrays in the canonical order. Now choosing $m$ requires searching $I_{k}$ for the minimum remaining column number in current order, and since this must be done as a linear search, it will require $O\left(\left|I_{k}\right|\right)$ time on the average. In return for this searching, we can again form $I_{k} \cup I_{m}$ in $O\left(\left|I_{m}\right|+\left|I_{k}\right|\right)$ time independent of the amount of pivoting, since both $I_{m}$ and $I_{k}$ are in increasing order with respect to the canonical order.

\section{NUMERICAL RESULTS}

In this section we give numerical results obtained by programming the five implementations of Section 3 and running the programs on several test problems. The results here are not comprehensive, but the problems are realistic ones. The problem GS192 arose in the solution of a parabolic partial differential equation and was supplied by Professor Paul Saylor of the University of Illinois. The others were taken from a collection of test problems supplied by Dr. Iain Duff of ACM Transactions on Mathematical Software, Vol 4, No 4, December 1978 
the Atomic Energy Research Establishment at Harwell, England. Brief descriptions of the problems are given in Table I. (See Duff and Reid [4] for further discussion of the Harwell test problems.)

Experiments have shown that the storage and time costs of the algorithms discussed here may depend heavily on the initial row and column orders of $A$. Except for GS192, we chose to order the rows in order of increasing number of nonzero entries and to order the columns symmetrically. For GS192, we used the matrix exactly as supplied to us. If the permutation matrix $P$ accomplishes the desired row reordering, then we applied the algorithms to the permuted system.

$$
P A P^{\mathrm{T}}(P x)=P b \text {. }
$$

There is little theoretical reason to expect that the choice of $P$ would have a significant effect on the accuracy of the computed solution $x$, except that reducing the number of arithmetic operations performed should give a corresponding reduction in the roundoff error incurred. In practice, however, we found that particularly inefficient orderings of the rows led to poor accuracy, while orderings which were at least reasonably efficient gave uniformly good results. Unfortunately, at present we can say no more than this, but we hope to investigate further the problem of choosing the initial row and column orderings. (Note, however, that the simple row reorderings described above gave good numerical results for our test problems.)

Table I

\begin{tabular}{|c|c|c|c|}
\hline Problem & N & Application & Remarks \\
\hline ARC 130 & 130 & $\begin{array}{l}\text { Laser } \\
\text { Research }\end{array}$ & \\
\hline SHLO & 663 & $\begin{array}{l}\text { Linear } \\
\text { Programming }\end{array}$ & Lower triangular \\
\hline SHL200 & 663 & $\begin{array}{l}\text { Linear } \\
\text { Programming }\end{array}$ & \\
\hline SHL400 & 663 & $\begin{array}{l}\text { Linear } \\
\text { Programming }\end{array}$ & \\
\hline STRO & 363 & $\begin{array}{l}\text { Linear } \\
\text { Programming }\end{array}$ & Lower triangular \\
\hline STR200 & 363 & $\begin{array}{l}\text { Linear } \\
\text { Programming }\end{array}$ & \\
\hline STR400 & 363 & $\begin{array}{l}\text { Linear } \\
\text { Programming }\end{array}$ & \\
\hline GS192 & 192 & $\begin{array}{c}\text { Parabolic } \\
\text { Partial } \\
\text { Differential } \\
\text { Equation }\end{array}$ & $\begin{array}{l}\text { Banded with half } \\
\text { bandwidth of } 19\end{array}$ \\
\hline
\end{tabular}

ACM Transactions on Mathematical Software, Vol 4, No. 4, December 1978 
To provide a benchmark comparison for our algorithms, we solved the test problems with a code due to Curtis and Reid [2] which is part of the Harwell Subroutine Library. This code uses a rather more sophisticated complete pivoting algorithm to factor $A$ and compute $x$. Where our algorithms preorder $A$ to exploit sparseness and then pivot entirely for numerical stability, the Harwell code uses a strategy known as threshold pivoting to do both at once.

In threshold pivoting the pivot element at the $k$ th step is chosen to be that entry $a_{\imath}, k \leq i, j \leq N$, which requires the fewest arithmetic operations to eliminate and which satisfies either

$$
\left|a_{\imath \jmath}\right| \geq u \cdot \max \left\{\left|a_{\imath l}\right|: k \leq l \leq N\right\} \quad \text { or } \quad\left|a_{\imath \jmath}\right| \geq u \cdot \max \left\{\left|a_{l \jmath}\right|: k \leq l \leq N\right\}
$$

where $u$ is a threshold parameter satisfying $0<u \leq 1$. For our experiments we used two different settings for $u: u=1.0 \mathrm{E}-6$, to allow pivoting almost entirely to exploit sparseness, and $u=1.0$, to force pivoting almost entirely for numerical stability. Note that the form of the threshold test allows for some flexibility in either case. For intermediate values of $u$, we would expect the code to exhibit a behavior somewhere between the two extremes we used.

Our experiments were run in single precision on an IBM System/360, Model 75 , using the Fortran IV level G compiler. All of the programs gave equally

Table II

\begin{tabular}{|c|c|c|c|c|c|c|c|c|c|}
\hline Method & & $\begin{array}{l}\text { Harwell } \\
u=1.0\end{array}$ & $\begin{array}{l}\text { Harwe } 11 \\
u=10^{-6}\end{array}$ & $\begin{array}{c}\text { Pun } \\
\text { Insertion }\end{array}$ & $\begin{array}{c}\text { Eubble } \\
\text { Sort }\end{array}$ & $\begin{array}{c}\text { Insertion } \\
\text { Sort }\end{array}$ & $\begin{array}{l}\text { She11 } \\
\text { Sort }\end{array}$ & $\begin{array}{c}\text { Heap } \\
\text { Insertion }\end{array}$ & $\begin{array}{l}\text { Pivot } \\
\text { Search }\end{array}$ \\
\hline \multirow{2}{*}{$\operatorname{ARC} 130$} & $N Z$ & 1293 & 1293 & 1457 & 1457 & 1457 & 1457 & 1457 & 1457 \\
\hline & Secs & 6.89 & 7.36 & 0.48 & 0.56 & 060 & 0.64 & 0.58 & 070 \\
\hline \multirow{2}{*}{ SHLO } & $\mathrm{NZ}$ & 1687 & 1687 & 2568 & 2568 & 2568 & 2568 & 2568 & 2568 \\
\hline & Secs. & 5.90 & 7.09 & 1.38 & 0.99 & 1.13 & 1.10 & 4.59 & 2.11 \\
\hline \multirow{2}{*}{ SHL200 } & $N Z$ & 1726 & 1726 & 2637 & 2637 & 2637 & 2637 & 2637 & 2637 \\
\hline & Secs. & 6.47 & 6.31 & 1.18 & 1.12 & 113 & 1.23 & 4.50 & 2.21 \\
\hline \multirow{2}{*}{ SHL400 } & $N Z$ & 1712 & 1712 & 2651 & 2651 & 2651 & 2651 & 2651 & 2651 \\
\hline & Secs & 6.36 & 7.09 & 119 & 1.08 & 1.04 & 1.17 & 434 & 2.16 \\
\hline \multirow{2}{*}{ STRO } & $N Z$ & 2454 & 2454 & 2953 & 2953 & 2953 & 2953 & 2953 & 2953 \\
\hline & Secs. & 3.83 & 4.49 & 0.61 & 0.48 & 050 & 0.48 & 1.90 & 0.73 \\
\hline \multirow{2}{*}{ STR200 } & $N Z$ & 3640 & 3516 & 7254 & 7254 & 7254 & 7254 & 7254 & 7254 \\
\hline & Secs. & 8.91 & 8.22 & 204 & 3.32 & 348 & 4.61 & 3.48 & 4.61 \\
\hline \multirow{2}{*}{ STR400 } & $N Z$ & 3879 & 3735 & 8233 & 8233 & 8233 & 8233 & 8233 & 8133 \\
\hline & Secs. & 10.91 & 9.58 & 2.43 & 3.45 & 3.82 & 5.14 & 3.89 & 4.35 \\
\hline \multirow{2}{*}{ GST92 } & $N Z$ & 6568 & $6746^{\star}$ & 6880 & 6880 & 6880 & 6880 & 6880 & 6880 \\
\hline & Secs. & 60.92 & $82.42^{\star}$ & 1022 & 14.41 & 19.97 & 35.79 & 10.57 & 17.95 \\
\hline
\end{tabular}

* The method farled to compute an accurate solution. 
accurate results as measured by the $L_{2}$ and $L_{\infty}$ norms of both the absolute error in the solution and the residual, except that the Harwell code with $u=1.0 \mathrm{E}-6$ was sometimes less accurate than the others. Hence the important question in our tests was efficiency, and we chose to use two measures: the number of nonzeros in the computed $L U$ factorization (as some measure of storage cost), and the time required to compute the solution $x$ from the original (not yet preordered) system (1.1). We should note that probably all of the codes could be made somewhat faster by more careful coding and that the times may be in error by approximately 10 percent due to system overhead. However, the results are still qualitatively interesting.

The results shown in Table II show several things. Most obvious, the partial pivoting codes are invariably much faster than the Harwell code, even though they produce a denser factorization (and hence perform more arithmetic operations). Next, it appears that the Shell Sort, Heap Insertion, and Pivot Search algorithms performed worse than the other partial pivoting algorithms on most of the problems. In the first case this is probably due to the nature of the data which must be sorted; in the second case, this is probably due to data structure overhead; and in the last case this is probably due to the fact that the costly searches occurred in the filled-in rows at each step. Finally, it seems that the four remaining partial pivoting algorithms all performed comparably well, with the Run Insertion algorithm appearing best over all, perhaps because it can be implemented with little programming overhead.

\section{CONCLUSION}

There seem to be several directions for future research in this area. On the theoretical side, there is a particularly troubling problem which may be stated as follows. Let $C(N)$ be the cost (in time) of solving a sparse system (1.1) without pivoting. (Assume for a moment that this is numerically stable.) It would be desirable for a pivoting algorithm to have a cost which was $O(N \cdot C(N))$ in the worst case and which declined to $C(N)$ as the amount of pivoting decreased. Unfortunately, none of the algorithms discussed here has both properties, and, in fact, it is unclear whether any algorithm could have them.

In a more practical vein, it is desirable to continue the testing and comparison program begun in the work reported in this paper. One problem of importance is the selection of the initial row ordering. Another question which might be investigated is whether there is some threshold technique which might be applied to partial pivoting as discussed here. Since no information is kept about the column structures of $A, L$, and $U$, the strategy used in the Harwell code is not suitable, but it might be possible to use a threshold parameter to decide whether or not to pivot at all in a certain row. Reducing the amount of pivoting in this way might make the algorithms run faster without significantly affecting accuracy.

Finally, we must turn to the eventual goal of work such as ours, the production of useful mathematical software. It is true that the behavior of a partial pivoting code depends quite heavily on the specific problem being solved, in particular on the amount of pivoting required and on the initial ordering of the rows and columns of $A$. Hence our sample of problems may provide a biased and incorrect 
view of the true situation. However, we believe that our results do provide enough encouragement to justify the development and extensive testing of high quality subroutines based on one or more of our algorithms; one based on the Run Insertion algorithm appears in the accompanying Algorithm 533.

\section{REFERENCES}

1 Aнo, A, Hopcroft, J.E, AND Ullman, J.D. The Design and Analysis of Computer Algortthms Addison-Wesley, Reading, Mass., 1974.

2. CuRTis, A.R., AND REID, J.K. FORTRAN subroutines for the solution of sparse sets of linear equations. AERE Rep. R6844, AERE Harwell, England, 1971.

3 CURTIS, A.R, AND REID, J.K The solution of large sparse unsymmetric systems of linear equations. Information Processing '71 (1971), 1240-1245.

4. DUFF, I.S., AND REID, J.K A comparison of sparsity orderings for obtaining a pivotal sequence in Gaussian elimination. AERE Rep. T.P. 526, AERE Harwell, England, 1973

5. EisENSTAT, S.C., AND ShERMAN, A.H Efficient implementation of sparse nonsymmetric Gaussian elımination whthout pivoting SIGNUM Newsletter 10 (1975), 26-29.

6. Forsythe, G.E., ANd Moler, C B Computer Solution of Linear Algebralc Systems PrenticeHall, Englewood Cliffs, N J , 1967

7 KNUTH, D.E. The Art of Computer Programming, Vol 3. Searching and Sortıng AddisonWesley, Reading, Mass., 1973.

Received July 1976; revised November 1977 